\title{
BMJ Quality Quality improvement: The delivery of true early mobilisation in an intensive care unit
}

\author{
Zoe van Willigen, Nikki Collings, Dominic Richardson, Rebecca Cusack
}

To cite: van Willigen $Z$, Collings N, Richardson D, et al. Quality improvement: The delivery of true early mobilisation in an intensive care unit. BMJ Quality Improvement Reports 2016;5:u211734.w4726 doi:10.1136/bmjquality. u211734.w4726

Received 29 July 2016 Revised 9 September 2016

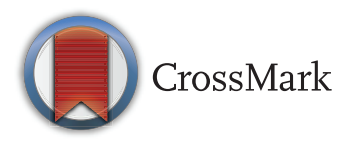

University Hospital Southampton NHS Foundation Trust, UK

Correspondence to Zoe van Willigen zomatt@hotmail.com

\section{ABSTRACT}

Early mobilisation initiatives within the critical care environment have been shown to improve outcomes for patients. Early mobilisation has been defined as occurring within the first two to five days of the intensive care stay, but in practice this can be difficult to deliver.

We conducted a quality improvement (QI) project to deliver early mobilisation in a large general intensive care unit. Mechanically ventilated medical patients received an integrated package of care involving two additional daily sessions of mobility therapy, in combination with minimal sedation where possible.

Prospective baseline data was collected from January to March 2012; the QI project commenced in April 2012. Improvement cycle 1 completed in March 2015 and improvement cycle 2 in March 2016. Results have suggested a reduction in time to first mobilisation for intensive care survivors from 16.3 days in 2012, to 4.3 days at the end of improvement cycle 2 . This was associated with a decrease in mean intensive care length of stay from 20.8 days in 2012, to 11.2 days at the end of improvement cycle 2.

This QI project enabled patients to mobilise out of bed within the first five days of their intensive care stay and to be discharged earlier from the ICU, on going analysis is required to verify these findings.

\section{PROBLEM}

Survival following critical illness is improving but is often associated with poor functional outcome, which may persist for years ${ }^{1}$. Rapid muscle wasting is observed in critically ill patients from the very early stages ${ }^{2}$ and is associated with the development of intensive care unit-acquired weakness (ICUAW). ${ }^{3}$ These adverse outcomes are associated with deep sedation, prolonged mechanical ventilation, severe sepsis and multi-organ dysfunction. ${ }^{45}$

There is growing expert consensus that early mobilisation on the intensive care unit (ICU) is beneficial ${ }^{6}$, with reported reductions in ventilator days and length of $\operatorname{stay}^{7} 8$ and improvements in functional outcome ${ }^{9}$. Hodgson et al. ${ }^{10}$ offer a definition that early mobilisation occurs within the first 2-5 days of the intensive care stay, involving mechanically ventilated patients performing volitional active exercise. However, recent articles report a wide range of timeframes from initiation within the first 72 hours of admission ${ }^{11}$, to any time point during mechanical ventilation on intensive care $^{8}$. In practice early mobilisation can be difficult to deliver requiring additional staff time, specialist equipment, and a co-ordinated team approach. ${ }^{12}$

University Hospital Southampton Foundation Trust (UHSFT) has a mixed medical and surgical adult GICU, admitting 1600 patients a year. GICU has 25 beds with nursing: patient ratios of 1:1 for Level 3 patients and 1:2 for Level 2 patients (levels are described in table 2). Prior to the QI project, physiotherapy staffing on GICU consisted of a 0.8 whole time equivalent (WTE) band 7, 2.5 WTE band 6 and 2.0 WTE band 5 qualified physiotherapists.

\section{BACKGROUND}

A systematic review evaluating the efficacy of specific interventions to improve physical function of ICU survivors concluded that the only effective intervention to improve longterm physical function is exercise/physical therapy, and that its benefit may be greater if started earlier ${ }^{13}$. Several studies have shown that early mobilisation initiatives within the critical care environment improve outcomes for both the patient and the wider health economy ${ }^{14-19}$. A randomised controlled trial demonstrated that the combined effects of early physical activity and reduced sedation is safe, well tolerated and results in less delirium, more ventilator free days and with better functional outcomes at hospital discharge. ${ }^{11}$

We set up a QI project targeted at delivering a similar early mobilisation programme in a large teaching hospital general intensive care unit. The aims of this project were to 
deliver early mobilisation and evaluate the impact on ICU length of stay, over a four-year period.

\section{BASELINE MEASUREMENT}

The project started in 2012 by measuring mobilisation activity within our general intensive care unit (GICU). Patients were enrolled on the project if they had been ventilated for less than 72 hours and were expected to remain ventilated for at least a further 24 hours. Patients were required to be cognitively intact and functionally independent (with or without a mobility aid) prior to admission. Patients were excluded if they were under 18 years of age, had rapidly deteriorating neuromuscular disease, raised intracranial pressure or were post cardiac arrest (until good neurological recovery could be confirmed). Patients with a body-mass index (BMI) of more than 35 were also excluded due to the weight restrictions of the rehabilitation equipment. Annual demographic and clinical admission data for patients enrolled in the QI project is shown in table 1 .

In order to address the aims of this project, data were collected regarding first out of bed mobilisation (sitting on edge of bed or out of bed activity) and ICU length of stay. We also collected number of therapy sessions (each session lasting on average 30 minutes), hospital length of stay, duration of ventilation and ventilator free days (in the first 28 days).The same data was collected prospectively in January-March for each year of the QI project. Data are reported for ICU survivors only. Mortality may affect length of stay and duration of ventilation for reasons not attributable to the QI intervention; for this reason only the ICU survivor group were selected for analysis. Baseline data demonstrated that patients were first mobilised out of bed in the pre QI period after 16.3 days and the mean length of stay for ICU survivors at that time was $20.8(+/-15.5)$ days.

\section{DESIGN}

A senior QI team of two consultant intensivists, one band 7 nurse and one band 7 physiotherapist was established to lead the early mobilisation project. Two new WTE band 4 therapy support worker posts were created to work alongside the existing therapy team to assist in the delivery of early mobilisation. A specific induction period was required to build expertise within the therapy team and train the therapy support workers for their role on GICU.

Prior to intervention, patients were assessed for medical stability with cardiorespiratory parameters required to be within a pre-defined range: heart rate $>40$ and $<130$ beats per minute; normal electrocardiogram; mean arterial pressure $>60 \mathrm{mmHg}$ and systolic blood pressure $<180 \mathrm{mmHg}$ with no increased vasopressor support in the preceding four hours; respiratory rate $<40$ breaths per minute; oxygen saturation $>90 \%$. Patients who were persistently agitated or combative and therefore unable to receive rehabilitation within the early time frame, were excluded from the project.

Patients received twice-daily 30-minute sessions of rehabilitation therapy in addition to standard physiotherapy sessions (usual care) for at least five days per week. This was in conjunction with the introduction of a new sedation and analgesia protocol ensuring minimal sedation where possible. Mobility therapy was started within 72 hours of the patient being intubated and ventilated, and was continued until discharge from ICU.

Table 1 Demographic and clinical admission data for ICU survivors enrolled in the QI project (Pre-QI data is included for comparison) $^{\mathrm{a}}$

\begin{tabular}{|c|c|c|c|c|c|}
\hline \multirow[b]{2}{*}{ January to March } & \multirow{2}{*}{$\begin{array}{l}\text { Pre-QI } \\
2012 \\
(n=16)\end{array}$} & \multicolumn{3}{|c|}{ Improvement Cycle 1} & \multirow{2}{*}{$\begin{array}{l}\text { Improvement Cycle } 2 \\
2016 \\
(n=19)\end{array}$} \\
\hline & & $\begin{array}{l}2013 \\
(n=19)\end{array}$ & $\begin{array}{l}2014 \\
(n=24)\end{array}$ & $\begin{array}{l}2015 \\
(n=22)\end{array}$ & \\
\hline Age (years) & $63.8(9.0)$ & $55.8(13.2)$ & $59.3(17.3)$ & $60.5(12.8)$ & $59.7(15.0)$ \\
\hline Gender (M:F), $n$ & $8: 8$ & $11: 8$ & $14: 10$ & $16: 6$ & $10: 9$ \\
\hline BMI & $26.6(7.8)$ & $25.9(9.2)$ & $25.5(5.1)$ & $27.1(4.6)$ & $23.5(3.4)$ \\
\hline APACHE II score & $23.2(5.7)$ & $20.1(6.7)$ & $19.6(6.2)$ & $19.0(8.6)$ & $17.9(5.8)$ \\
\hline $\mathrm{CCl}$ & $1.6(0.9)$ & $0.9(1.1)$ & $1.3(2.1)$ & $0.9(1.0)$ & $1.0(1.7)$ \\
\hline Admission type (n (\%)) & $\triangleright$ & $\triangleright$ & $\triangleright$ & $\triangleright$ & $\triangleright$ \\
\hline - Respiratory medicine & - $16(100 \%)$ & - $14(74 \%)$ & - $18(76 \%)$ & - $14(65 \%)$ & - $10(53 \%)$ \\
\hline - Renal medicine & $>0$ & $>0$ & - $1(4 \%)$ & - $1(4 \%)$ & - $1(5 \%)$ \\
\hline - Hepatology & $>0$ & - $4(21 \%)$ & - $2(8 \%)$ & - $1(4 \%)$ & - $1(5 \%)$ \\
\hline - Gastroenterology & $\rightarrow 0$ & $>0$ & $>0$ & $>0$ & - $2(11 \%)$ \\
\hline - Cardiology & $>0$ & - $1(5 \%)$ & - $2(8 \%)$ & - $5(23 \%)$ & - $4(21 \%)$ \\
\hline - Haemotology & $>0$ & $>0$ & $>0$ & - $1(4 \%)$ & $>0$ \\
\hline - Trauma & $>0$ & $>0$ & - $1(4 \%)$ & $>0$ & - $1(5 \%)$ \\
\hline - Surgical & $>0$ & $>0$ & $>0$ & $>0$ & $>0$ \\
\hline
\end{tabular}

${ }^{a}$ BMI: Body Mass Index. APACHE II: Acute Physiological and Chronic Health Evaluation II (20). CCI: Charlson Comorbidity Index (21), ICU: Intensive Care Unit. (APACHE II score reflects the severity of disease on admission to intensive care. $\mathrm{CCl}$ indicates the burden of existing comorbidities).

Data are expressed as mean (standard deviation), unless otherwise indicated. 
Table 2 Classifications of level of clinical care provided to patients

\section{Level Classification}

o Patients whose needs can be met through normal ward care in an acute hospital.

1 Patients at risk of their condition deteriorating, or those recently relocated from higher levels of care whose needs can be met on an acute ward with additional advice and support from the critical care team.

2 Patients requiring more detailed observation or intervention including support for a single failing organ system or postoperative care, and those stepping down from higher levels of care.

3 Patients needing monitoring and support for two or more organ systems one of which may be basic or advanced respiratory support.

From Comprehensive Critical Care, DH, 2000 (22)

\section{STRATEGY}

Quality Improvement Process

The structured QI model "The Four Es"; Engage, Educate, Execute and Evaluate $^{23}$ was the conceptual strategy for change employed to implement our QI project. This approach has been used in similar quality improvement projects as a means of translating evidence based interventions into practice. ${ }^{14} 16$ The approach involves creating a lead team, understanding the problem, enlisting relevant stakeholders and creating a change in practice.

Improvement cycle 1 - April 2012 to March 2015

We engaged and educated the multi-disciplinary team (MDT) on GICU by promoting the relevant literature on early rehabilitation, and holding informal focus groups with key members. The focus groups concentrated on discussing the size of the problem and potential solutions, and consideration was given to potential barriers to implementing change highlighted by the group. A multi-faceted education program was introduced for all GICU therapy, nursing and medical staff. One-to-one bedside teaching, single discipline and MDT group sessions, and demonstration using a YouTube video were employed. ${ }^{24}$ Patients were enrolled in the QI project and the early mobilisation intervention was executed. Outcomes were evaluated via a three-month audit conducted between January and March each year. Data were collected throughout the entire QI period, however due to staffing time constraints only the period of January to March was selected annually for thorough analysis and evaluation. Action at the end of cycle 1 was to re-engage new and existing members of the GICU MDT and to provide a more comprehensive education package for staff.

Improvement cycle 2 - April 2015 to March 2016

In order to re-engage the MDT the existing education programme was introduced on a rolling basis due to the high turnover of rotational staff on GICU. Additional education in the assessment of sedation and delirium was provided to assess patient's readiness for rehabilitation. Prompt cards outlining the sedation protocols and for the Richmond Agitation and Sedation Score (RASS) $^{25}$ and the Confusion Assessment Method for the Intensive Care Unit (CAM-ICU) ${ }^{26}$ were produced. We also introduced a competence-based learning package to formalise training and re-engage the therapy team. Finally, further execution of the project continued with daily therapy ward rounds (to ensure patients had a plan each morning), weekly MDT ward rounds and monthly MDT meetings to emphasise the collaborative approach of the early mobilisation programme. They were used to highlight any barriers experienced at the bedside and discuss potential solutions, to ensure patient progress within the project was not impeded. Data were again analysed for the period of January to March 2016 in order to re-evaluate the outcomes of the project.

\section{RESULTS}

A total of 112 patients were included in the QI data collection periods from 2013 to 2016; 84 (75\%) of these patients survived until ICU discharge.

Baseline data (January to March 2012) showed that patients surviving to ICU discharge were first mobilised out of bed, on average, after 16.3 days and had a mean length of ICU stay of 20.8 days. After improvement cycle 1 (April 2012 to March 2015), early mobilisation improved; patients mobilised out of bed, on average, 8.3 days earlier, and this was associated with a reduction in mean ICU length of stay by 6.6 days.

Analysis within improvement cycle 1 showed fluctuations in time to first mobilisation and ICU length of stay, with the results in 2014 not sustained into 2015 (Table 3). It became apparent that with junior team staff turn over, the momentum of the initial improvement had slowed, highlighting a necessity for re-education and more advanced methods of engagement and education. Following the refinements made in improvement cycle 2 positive results were regained; patients achieved out of bed mobilisation, on average, 4.3 days after admission, satisfying Hodgson's definition of early mobilisation ${ }^{10}$. This was associated with a further reduction in mean ICU length of stay by 3.0 days following improvement cycle 2 .

Secondary outcomes showed similar trends throughout both improvement cycles. The mean number of therapy sessions received by ICU survivors doubled following improvement cycle 1 and this was sustained during improvement cycle 2. Hospital length of stay decreased, on average, by 11.9 days following improvement cycle 1 and by a further 3.9 days following improvement cycle 2 (graph 1)

\section{LESSONS AND LIMITATIONS}

\section{Lessons}

Overall the time to first rehabilitation and intensive care length of stay results were much improved following 
Table 3 Clinical outcome data for ICU survivors enrolled in the QI project (Pre-QI data is included for comparison)b

\begin{tabular}{|c|c|c|c|c|c|}
\hline \multirow[b]{2}{*}{ January to March } & \multirow{2}{*}{$\begin{array}{l}\text { Pre-QI } \\
2012 \\
(n=16)\end{array}$} & \multicolumn{3}{|c|}{ Improvement Cycle 1} & \multirow{2}{*}{$\begin{array}{l}\text { Improvement Cycle } 2 \\
2016 \\
(n=19)\end{array}$} \\
\hline & & $\begin{array}{l}2013 \\
(n=19)\end{array}$ & $\begin{array}{l}2014 \\
(n=24)\end{array}$ & $\begin{array}{l}2015 \\
(n=22)\end{array}$ & \\
\hline Time to first out of bed mobilisation (days) & $16.3(10.9)$ & $9.3(8.3)$ & $6.4(4.1)$ & $8.0(4.7)$ & $4.3(2.7)$ \\
\hline ICU length of stay & $20.8(15.5)$ & $18.9(19.5)$ & $13.0(9.6)$ & $14.2(8.5)$ & $11.2(7.7)$ \\
\hline No. of therapy sessions, per day per patient & $0.4(0.2)$ & $1.1(0.4)$ & $1.2(0.4)$ & $0.8(0.4)$ & $0.8(0.3)$ \\
\hline Hospital length of stay & $45.4(50.4)$ & $36.9(28.3)$ & $30.2(23.7)$ & $33.5(32.2)$ & $29.6(18.4)$ \\
\hline Duration of mechanical ventilation (days) & $15.8(14.6)$ & $13.8(16.0)$ & $7.3(6.9)$ & $7.9(6.2)$ & $8.7(6.1)$ \\
\hline Ventilator-free days (within first 28 days) & $14.4(9.9)$ & $17.1(9.3)$ & $20.7(6.9)$ & $20.2(6.2)$ & $19.3(6.1)$ \\
\hline
\end{tabular}

Graph 1 Main clinical outcomes for ICU survivors enrolled in the QI project (Pre-QI data is included for comparison) ${ }^{\mathrm{C}}$

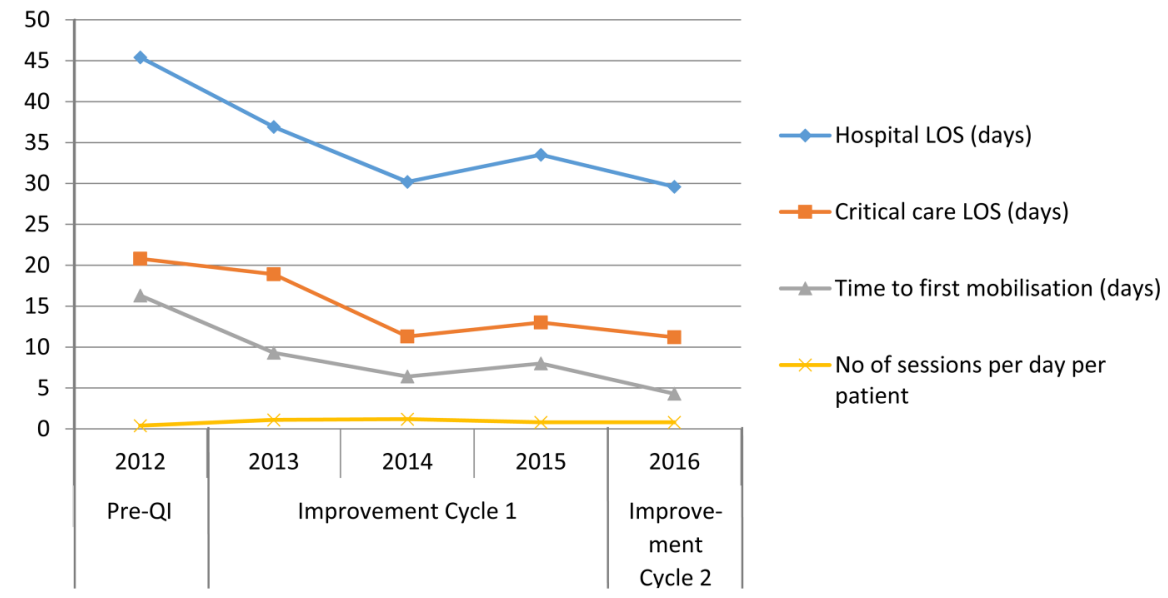

'LOS : Length of stay Data are displayed as means.

improvement cycle 1 . However, the results in 2014 were not quite sustained in 2015. This was attributed to staff turnover with less emphasis on education of the incoming staff in 2015. Actions taken from improvement cycle 1 included an enhanced education package and regular MDT ward rounds to re-emphasize the aims and objectives of the QI project.

Following improvement cycle 2 the renewed engagement of multi-disciplinary members contributed to the project's ongoing success. Weekly MDT ward rounds supported good communication and promoted a collaborative team approach within our ICU and were key to the sustained change in culture that we now observe on our unit.

We acknowledge the achieved number of therapy sessions is lower than the planned additional two sessions per day, as set out in the original protocol. Reasons for not delivering two additional daily sessions were not formally collected during the project, but anecdotally, barriers included physiological instability of the patient, patient receiving medical interventions and inadequate staffing levels.

It has been highlighted in the literature that a lack of resources and specialist equipment is a barrier to the provision of early mobilisation programs ${ }^{12}$. Our QI project was only possible due to initial investment for expansion of the therapy team and new equipment. Reducing intensive care length of stay can result in large cost savings for a hospital trust, in addition to increasing bed capacity for ICU admissions; this demonstrates that investment into similar QI interventions in other trusts may be cost effective.

Limitations

We acknowledge that there are limitations of our QI project. The before-after design and lack of blinding of the study team are weaknesses and may have subjected the results to bias. Our results suggest that the QI intervention had an impact on time to first mobilisation and length of ICU stay.

Our data was collected over 3 months on an annual basis such that we have 5 data points. Data could only be collected for 3 months every year due to limited resources. As a result, there may have been fluctuations in the data over throughout the remaining 9 months of each year, and there is the possibility that our results could be due to chance. If the project was to be repeated we would plan to analyse data throughout the year and at more frequent time intervals. 
Clinically, early mobilisation is not possible without minimal sedation; our quality improvement project involves the two interventions being used concurrently to improve outcomes for a group of mechanically ventilated medical patients. It is unlikely that either individual component of the intervention would have had similar results in isolation. However, initiating two changes simultaneously makes it difficult to clarify which change had the bigger impact. The 4 Es approach was used for this QI project as it recognises the value of engaging staff and culture change. Future work should consider using plan-do-study-act cycles alongside the 4 Es to allow testing of individual interventions where possible prior to implementation.

It should be noted that no other major QI projects were introduced during this time period, and consultant medical and senior nursing staff remained consistent. The results are encouraging within our cohort of patients. Caution should be taken in generalisation of these results to the wider ICU patient population, due to the heterogeneous nature of these patients and differences in local service provision. The data collected during this project is associated with large standard deviations due to the significant variability in length of stay that is commonly found in ICU patient populations. In view of this both mean and medians were calculated for each individual parameter. On review, the mean and median results were comparable and for the purposes of comparison with other projects published in the literature, it was agreed that reporting mean results only was most appropriate. A larger sample size may have reduced the variability of our results, but a detailed analysis was only possible for three months of data each year within the limited resources available to this project.

This QI project excluded patients with a BMI of more than 35 due to the weight restrictions of our rehabilitation equipment. This meant that the progressive design of the therapy interventions could not be used for these patients. Patients with a BMI of more than 35 do receive rehabilitation on our GICU, but for the aforementioned reason could not be included in our analysis.

\section{CONCLUSION}

We have demonstrated that the use of a structured quality improvement process is an effective means of generating changes which suggest improved outcomes for patients in our general intensive care unit. We have achieved our project aims and shown that early mobilisation and rehabilitation, delivered by a dedicated therapy team and supported by the MDT, achieves true early mobilisation and reduces ICU length of stay, however on going more detailed analysis, is required to verify this. These improvements have been sustained by prioritising engagement with ICU staff, supported with a comprehensive educational programme.

Early rehabilitation for mechanically ventilated patients on the ICU is currently receiving much attention and is associated with improved physical function and quality of life outcomes for patients following critical illness. Few centres achieve Hodgson's definition of early mobilisation within 2-5 days ${ }^{10} 1627$. Our QI project has enabled patients to mobilise out of bed within the first five days of their intensive care stay and to be discharged earlier from the ICU.

Acknowledgements The assistance of the dedicated staff at University Hospital Southampton Foundation Trust General Intensive Care Unit is gratefully acknowledged.

In particular: Leanne Gallagher, Victoria Mulligan, Shirley Austin, Danielle Close-Brooke, Jessica Swinburne and Marina Stanevska.

The authors thank Dr Lisa Roberts for her help in proof reading this quality improvement project.

The authors would also like to acknowledge the help and financial support of Health Education Wessex.

The cost of the quality improvement project was funded by University Hospital Southampton Foundation NHS Trust Innovation Fund.

\section{Declaration of interests Nothing to declare}

Ethical approval This piece of work reports the prospective audit data collected over a four year period during which the Trust funded an Innovation project to improve the delivery of physiotherapy services to patients within the Intensive Care Unit at Southampton University Hospital. The effect of the introduction of this project was monitored with a yearly, 3 month audits (Jan to March; 2012-2016). These audits were registered with the Clinical Effectiveness Department at the University Hospital of Southampton NHS FT (Trust Audit Ref ZAUD2451).

According to the policy of University Hospital of Southampton NHS FT this work met the criteria for operational improvement and as such is exempt from ethics review.

Open Access This is an open-access article distributed under the terms of the Creative Commons Attribution Non-commercial License, which permits use, distribution, and reproduction in any medium, provided the original work is properly cited, the use is non commercial and is otherwise in compliance with the license. See:

- http://creativecommons.org/licenses/by-nc/2.0/

- http://creativecommons.org/licenses/by-nc/2.0/legalcode

\section{REFERENCES}

1. Herridge MS, Tansey CM, MattC A et al. Functional disability 5 years after acute respiratory distress syndrome. N Engl J Med 2011; 364:1293-304.

2. Puthucheary ZA, Rawal J, McPhail M et al. Acute skeletal muscle wasting in critical illness. JAMA 2013; 310:1591-600.

3. Fan E, Cheek F, Chian L et al. An official American Thoracic Society Clinical Practice Guideline: a diagnosis of intensive care unit-acquired weakness in adults. Am J Resp Crit Care Med 2014; 190:1437-46.

4. De Jonghe B, Sharshar T, Lefaucheur J-P et al. Paresis acquired in the intensive care unit: a prospective mulitcentre study. Journal of the American Medical Association 2002; 288, 2859-2867.

5. Stevens RD, Dowdy DW, Michaels RK, Mendez-Tellez PA, Pronovost PJ, Needham DM. Neuromuscular dysfunction acquired in critical illness: a systematic review. Intensive Care Medicine 2007; 33, 1876-1891.

6. Hodgson C, Stiller K, Needham DM et al. Expert consensus and recommendations on safety criteria for active mobilization of mechanically ventilated critically ill adults. Critical Care 2014; 18:658.

7. Gosselink R, Bott J, Johnson M, Dean RE, Nava S, Norrenberg M. Physiotherapy for adults with critical illness: recommendation of the European Respiratory Society of Intensive Care Medicine Task Force on Physiotherapy for Critically III Patients. Int Care Med 2008; 34: 1188-1199.

8. Kayambu G, Boots R, Paratz J. Physical therapy for the critically ill in the ICU. Crit Care Med 2013; 41: 1543-1554.

9. Adler J, Malone D. Early mobilisation in the intensive care unit: a systematic review. Cardiopulm Phys Ther J 2012; 21: 5-13. 
10. Hodgson C, Bellomo R, Berney S, et al. for The TEAM Study Investigators. Early mobilization and recovery in mechanically ventilated patients in the ICU: a bi-national, multi-centre, prospective cohort study. Critical Care 2015; 19: 81-90.

11. Schweickert WD, Pohlman M, Pohlman A, Nigos C, Pawlik A, Esbrook C. Early physical and occupational therapy in mechanically ventilated, critically ill patients: a randomised controlled trial. Lancet 2009; 373:1874-82.

12. Hodgson CL, Berney S, Harrold M, Saxena M, Bellow R. Clinical review: early patient mobilization in the ICU. Critical Care 2013; 17: 207.

13. Calvo-Ayala E, Khan BA, Farber MO, Ely EW, Boustani MA Interventions to Improve the Physical Function of ICU Survivors. Chest 2013; 144: 1469-1480.

14. Needham DM, Korupolu R, Zanni JM, Pradhan P, Colantuoni E, Palmer JB. Early physical medicine and rehabilitation for patients with acute respiratory failure: a quality improvement project. Arch Phys Med Rehabil 2010; 91:536-42.

15. Morris PE, Goad A, Thompson C, Taylor K, Harry B, Passmore L. Early intensive care unit mobility therapy in the treatment of acute respiratory failure. Crit Care Med 2008; 36:2238-243.

16. McWilliams D, Weblin J, Atkins $G$ et al. Enhancing rehabilitation of mechanically ventilated patients in the intensive care unit: A quality improvement project. Journal of Critical Care 2015;30: 13-18.

17. Bailey P, Thomsen GE, Spuhler VJ, Blair R, Jewkes J, Bezdjian L. Early activity is feasible and safe in respiratory failure. Crit Care Med 2007;35:139-145.

18. Sricharoenchai T, Parker AM, Zanni JM, Nelliot A, Dinglas VD, Needham DM. Safety of physical therapy interventions in critically ill patients: a single-center prospective evaluation of 1110 intensive care unit admissions. J Crit Care 2014;29:395-400.

19. Lord RK, Mayhew CR, Korupolu R et al. ICU Early Physical rehabilitation programs: Financial modeling of cost savings. Crit Care Med 2013;41:717-724.

20. Knaus WA, Draper EA, Wagner DP, Zimmerman JE. APACHE II: a severity of disease classification system. Crit Care Med 1985;13:818-829.

21. Charlson ME, Pompei P, Ales KL, MacKenzie CR. A new method of classifying prognostic comorbidity in longitudinal studies: development and validation. J Chronic Dis 1987;40:373-83.

22. Department of Health. Critical care outreach 2003: progress in developing services. Department of Health and Modernisation Agency, 2003.

23. Pronovost PJ, Berenholtz SM, Needham DM. Translating evidence into practice: a model for large scale knowledge translation. BMJ 2008;337:1714.

24. CAM-ICU Delirium Test - YouTube. www.youtube.com/watch? $\mathrm{v}=6 \mathrm{WyJ}$ OzL7Vk

25. Ely EW, Truman B, Shintani A. Monitoring sedation status over time in ICU patients: reliability and validity of the Richmond Agitation-Sedation Scale (RASS). JAMA 2003;289: 2983-2991.

26. Ely EW, Inouye SK, Bernard GR et al. Delirium in mechanically ventilated patients: validity and reliability of the confusion assessment method for the intensive care unit (CAM-ICU). JAMA 2001; 286:2703-10.

27. Harrold ME, Salisbury LG, Webb SA, Allison GT. Early mobilisation in intensive care units in Australia and Scotland: a prospective, observational cohort study examining mobilisation practises and barriers. Critical Care 2015;19:336 